\title{
RESPONSABILIDAD SOCIAL EMPRESARIAL: ¿HERRAMIENTA PARA EL DESARROLLO DE LOS EMPRENDIMIENTOS?
}

\section{CORPORATE SOCIAL RESPONSIBILITY: TOOL FOR THE DEVELOPMENT OF THE ENTERPRISES?}

\author{
Lizbeth Suárez Morales \\ Universidad Internacional del Ecuador - UIDE \\ lsuarez@uide.edu.ec
}

RECIBIDO: 03 / 04 / 2017

APROBADO: 19 / 05 / 2017

\section{Resumen}

El objetivo del presente artículo, es analizar la RSE como una herramienta de desarrollo de los Emprendimientos, realizando un breve recorrido teórico por los paradigmas funcionalista e interaccionista, el primero que tiene raíces en las teorías positivistas y evolucionistas que han caracterizado históricamente el pensamiento científico de Occidente; y la segunda también llamada de conflicto, que se basa en las teorías críticas y libertarias enraizadas en el marxismo, el existencialismo, el anarquismo, la fenomenología, la teoría crítica y el enfoque de la acción humana.

La Responsabilidad Social Empresarial, como parte del paradigma interaccionista renueva la concepción de la empresa, otorgando a ésta una dimensión amplia e integradora, que va más allá de la mera cuestión económica en la que se incorpora perfectamente la triple faceta de la sostenibilidad: económica, social y medioambiental. El desarrollo sostenible se sitúa como fin a alcanzar por medio de la adecuada implantación de un modelo de empresa socialmente responsable, en el que los distintos grupos de interés, stakeholders, son el centro de atención esencial para la gestión. En el caso del Ecuador el $90 \%$ de las empresas proceden de emprendimientos familiares, que en casos citados se puede identificar que las empresas ecuatorianas, aplican RSE como una esta buena práctica para garantizar el desarrollo de los a emprendimientos socialmente responsables.

Palabras Clave: Responsabilidad Social Empresarial, Emprendimiento, Teoría funcionalista, Teoría interaccionista, stakeholders.

\begin{abstract}
The objective of this article is to analyze CSR as a tool for the development of Entrepreneurship in Ecuador, taking a brief theoretical tour of the functionalist and interactionist paradigms, the first that roots in the positivist and evolutionary theories that have historically characterized the scientific thinking of the West; and the second also called conflict that is based on critical and libertarian theories rooted in Marxism, existentialism, anarchism, phenomenology, critical theory and the focus of human action. Corporate Social Responsibility, as part of the interactionist paradigm, renews the company's conception, granting it a broad and inclusive dimension, which goes beyond the mere economic question that perfectly incorporates the threefold aspect of sustainability: economic, social and environmental. Sustainable development is a goal to be achieved through the adequate implementation of a socially responsible enterprise model, in which the different interest groups, stakeholders, are the essential focus for management. In the case of Ecuador, $90 \%$ of the companies come from family
\end{abstract}


enterprises, which in cases cited can be identified that Ecuadorian companies apply CSR as a good practice to ensure the development of socially responsible enterprises.

Key Words: Corporate Social Responsibility, Entrepreneurship, Functionalist theory, Interactionist theory, stakeholders.

\section{Metodología}

La metodología utilizada en este trabajo es descriptivo a través del análisis bibliográfico de bases de datos, se utilizó palabras clave de búsqueda "responsabilidad social empresarial y emprendimientos", de aquella búsqueda en total se encontraron 3831 artículos relacionados con la temática de investigación, que luego del análisis de contenido, se han identificado 6 publicaciones académicas en directa relación a la tema, subtema y título de este escrito.

\section{Discusión de Datos \\ Responsabilidad Social}

Para alcanzar el meollo de la Responsabilidad Social, se propicia la conceptualización del término "Responsabilidad, desde el punto de vista ético; todos somos responsables por todos", además desde la cualidad del concepto, se relaciona ampliamente con la misericordia (virtud) y la moral (doctrina). (Toca, 2017)

Referenciando la fundamentación de la Teoría Social de Coleman, sostiene, que el tejido social, como la construcción de interacciones entre agentes que se relacionan entre sí; ciudadanos, gobierno y actividades productivas en el marco económico dan paso a la sociedad.(Coleman, 1990).

Al fusionar los conceptos de Responsabilidad y lo Social, se avizora una significación que, ha "suscitado numerosos "debates" a cerca de la conveniencia o no de su aplicación, así como de las posibles ventajas que la integración en las políticas empresariales podrían causar". (Izquierdo \& Vicedo, 2009: 9)

El concepto de Responsabilidad Social, es un concepto moderno que cautiva a las empresas y sus directores en la búsqueda de incorporarse en las nuevas tendencias de negocios para un mejor desempeño, para ello es importante realizar algunas precisiones acerca del concepto de esta palabra.(Vives, 2013)

Retrotrayéndose en el tiempo, en América Latina, (Kliksberg, 2006), en su publicación, "Ética Empresarial: ¿Moda o demanda imparable?”, detalla tres etapas en la evolución de la idea de Responsabilidad Social por parte de los empresarios: la primera que se enfoca en el interés de las empresas de generar rentabilidad para los accionistas y lo que se espera de la empresa es que mejore la calidad de vida de los trabajadores, sus familias y la sociedad en general; la segunda etapa, caracterizada por la filantropía empresarial, donde priman las donaciones para actividades educativas y culturales anhelando que las empresas a más de entregar dinero, contribuyan a la defensa de las grandes causas sociales y la tercera, que conjuga un buen gobierno corporativo, el respeto a los consumidores, al medio ambiente y un compromiso social efectivo.

Se puede interpretar que estas etapas están constituidas por tres elementos importantes como: la conciencia ética del ser humano y su proceder cotidiano, la naturaleza del hombre como miembro de un grupo y un segmento económico en pleno desarrollo. 
Por otra parte (Friedman, 1966), manifiesta, "El que nuestros dirigentes de empresa aceptaran la idea de que les corresponde una responsabilidad social que no sea el obtener el mayor beneficio posible para sus accionistas sería tanto como socavar los cimientos de nuestra sociedad libre".

Entonces la RSE, no solo confirma la finalidad de producir y hacer riqueza, sino también el de brindar oportunidades de trabajo y bienestar a través de la incorporación de aquellas prácticas apegadas a la ética que mediante estrategias de las empresas o instituciones buscan mejorar el bienestar de la sociedad.

Para comprender el concepto de RSE, se debe profundizar en la raíz misma de los paradigmas de pensamiento, por ello se cita a (Benno, 1996), que habla de dos paradigmas, el primero "El funcionalismo y el segundo el interaccionismo en la gestión que representan dos tradiciones teóricas opuestas que tienen su fundamento en conceptos distintos de ser humano, en distintas teorías de sociedad, distintas filosofías de ciencia y distintas orientaciones pedagógicas".

\section{Paradigma Funcionalista}

Por una parte el Funcionalismo recopila los conceptos positivistas y evolucionistas originados por Comte, Spencer, Pareto y Durkheim, para abordar los más recientes, como Lewin, Homans, Merton y Parsons; estos y otros representantes influyentes del positivismo filosófico y del funcionalismo sociológico en las ciencias sociales son protagonistas de teorías administrativas caracterizadas por modelos hipotéticodeductivos, por la precisión, la lógica simbólica, el comportamiento funcional y la investigación empírica apoyada en la extensa utilización de los métodos de las ciencias naturales.

Dichas teorías de administración tienen por objetivo el logro del orden y del progreso social, la integración y la cohesión social, la satisfacción de las necesidades sociales y la reproducción estructural y cultural de la sociedad. Para alcanzar dichos objetivos de manera eficiente y eficaz, las teorías positivistas de administración enfatizan el orden y el equilibrio y tienden a ser realistas y racionalistas. Dichas teorías administrativas constituyen el llamado paradigma tradicional o utilitarista, que ha prevalecido en la investigación y la práctica de la gestión de Occidente durante más de medio siglo.

En la práctica administrativa se enfatizan tres dimensiones en el paradigma funcionalista, que corresponden a criterios predominantes de desempeño administrativo: la primera, es la administración burocrática, en la que la mediación es normativa, pues enfatiza la dimensión institucional del comportamiento organizacional; la segunda administración idiosincrática, en la que la mediación es personalista, pues enfatiza la dimensión individual; y la tercera la administración integradora, en la que la mediación es ambivalente, ya que enfatiza simultáneamente la dimensión institucional o individual. Es así que el paradigma funcionalista de la administración define una línea administrativa que fortalece la institucionalidad normativa, el comportamiento organizacional y el individualismo.

\section{Paradigma Interaccionista}

Para (Benno, 1996), reúne las teorías críticas y libertarias del conflicto en las ciencias sociales. 
Las primeras formulaciones de perspectivas alternativas de organización y gestión educativa protagonizadas por teóricos críticos de la tradición del conflicto en las ciencias sociales están ligadas al cuestionamiento de los fundamentos y supuestos positivistas y funcionalistas de la administración tradicional. En realidad, los teóricos críticos conciben la administración interaccionista del conflicto como antítesis de la administración funcionalista del consenso, argumentando que las perspectivas tradicionales de gestión no han sido capaces de ofrecer explicaciones suficientes y adecuadas acerca de los fenómenos del poder, la ideología, el cambio y las contradicciones que caracterizan el sistema educativo en el contexto de la sociedad contemporánea.

Finalmente (Benno, 1996), denota que los fundamentos iniciales de la administración interaccionista se hallan en la economía política de Marx, en el existencialismo de Kierkegaard y Sartre aliado al idealismo de Kant, Fichte y Hegel, en la fenomenología de Husserl y en el anarquismo de Proudhon. A la luz de esas corrientes intelectuales y de algunas propuestas progresistas de orientación liberal, como las de Dahrendorf, las teorías interaccionistas del conflicto tienen por objeto la concientización y la interpretación crítica de la realidad, el alcance de la emancipación humana y la transformación estructural.

El paradigma interaccionista permite realizar un análisis crítico de la realidad para realizar cambios estructurales en la forma de administrar las empresas de una forma crítica y analítica.

Entonces, se puede manifestar que la RSE es la evolución del pensamiento administrativo y pertenece al paradigma interaccionista en donde se puede comulgar con el manifiesto de (Kliksberg, 2006), que señala que la RSE es lo opuesto de una moda. Es parte de un proceso de evolución de la concepción misma de la empresa privada en las últimas décadas.

En efecto, la responsabilidad de todo ser humano, está en función de sus valores humanos y desde este panorama cualquier iniciativa de emprendimiento deberá estar fundamentada en el respeto.

Este proceso está movilizado por poderosas demandas sociales. Las mismas parten del reconocimiento de que la empresa privada es un motor esencial de la economía, y que sus actividades deben ser promovidas y facilitadas pero que, justamente por eso, por su enorme incidencia en la vida de los ciudadanos, tiene que ser considerada como una institución social con responsabilidades calificadas, y debe autoconsiderarse de este modo.

\section{Emprendimiento}

Para (Orrego, 2008), "El emprendimiento ha heredado una visión funcionalista de la administración, desde la cual, la mayoría de las entidades emprendedoras colocan su énfasis en recetas, llamadas en este caso "planes de negocios" estructurados en diferentes estudios y en el uso de una serie de procedimientos organizacionales, que impactan las nuevas propuestas de un alto índice de funcionalidad. El camino que se compadece con la concepción humana del emprendimiento es la solidaridad; desde la cual se propone la igualdad en la búsqueda de oportunidades y se rechaza toda posición de poder que ostente inclinaciones por actos de acumulación de riqueza". 
La débil estructura y la escaza disposición de recursos con los que empieza actividades, un emprendimiento merma la atención que el emprendedor le da a aquellos recursos que enmarcan su labor, como lo es la sociedad, la ética como un eje trasversal en las actividades empresariales de diversa magnitud, se ha intensificado en las últimas décadas, propicio el aparecimiento de la responsabilidad social que ha influenciado varios escenarios de la vida humana, con mayor intensidad se ha ocupado del ámbito empresarial; puesto que las organizaciones son constructos humanos y por ende su reflexión esta imbricada por una ética social.(Orrego, 2008)

Presupuestar el conjunto de obligaciones que se derivan de los impactos de la actividad empresarial en los ámbitos, social, laboral, económico y ambiental, implica para la responsabilidad social abordar la dimensión ética y en consecuencia establecer una relación directa con el componente axiológico de los miembros de la organización.

Para (Drakopoulou Dodd \& Hynes, 2011). El emprendedor, se considera una persona con ideas innovadoras, que desarrolla y comercializa en el mercado y que percibe una oportunidad y crea un nuevo negocio económico y social de una región.

Por otra parte, (Bilbao \& Pachano, 2002) manifiesta, "El emprendedor exitoso es una persona con un sueño, un objetivo, un deseo de crear, de innovar, que capta una oportunidad de negocio, que es capaz de 'ver' su idea en forma terminada, que no se detiene ante los obstáculos, por lo que la persistencia y la tenacidad con características típicas de su comportamiento. La mayoría de las veces emprende en un área de su especialidad, donde posee un conocimiento previo, donde le gusta trabajar. El emprendedor puede imaginar con gran precisión el resultado final y, aunque puede trabajar bajo un plan, no se detiene demasiado en pensar en el proceso.

Es por ello que los emprendimientos al ser creados bajo el paradigma funcionalista en donde prima la empresa como un entes, con fines de lucro, consumo, mercado, clientes y proveedores, se han convertido en unidades cerradas en las que no caben los términos éticos o de responsabilidad, que generen un cambio en la forma de hacer las cosas.

\section{La Responsabilidad Social como herramienta de desarrollo de los emprendimientos.}

La RSE desde su fundamentación ética, se ha identificado con el paradigma de Pensamiento Interaccionista, que converge en el análisis crítico de la realidad para realizar cambios estructurales en la forma de administrar las empresas de una forma crítica y analítica.

Desde una perspectiva aplicada (Druker, 2001), plantea las fronteras de la gerencia, y específicamente sostenía que la responsabilidad social empresarial debía integrar el núcleo duro de la gerencia, en tanto que las empresas son instituciones sociales basadas en las relaciones de confianza entre todos los actores involucrados y que sus activos más importantes eran el capital humano, el capital social y la reputación corporativa.

Por otra parte (Klilsberg, 2006) manifiesta que se debe asociar la responsabilidad empresarial con el capital social, en el marco de la recuperación expansiva y abarcadora de la conducta ética.

Adicionalmente Kofi Annan propone, que la RSE debe ser de carácter incluyente y concluyente, generando una propuesta de Pacto Global, que representa un documento de 
compromiso de las empresas y demás organizaciones privadas de la sociedad en favor del ejercicio de la ciudadanía social.

En virtud de lo antes citado las empresas y en este caso los emprendimientos además de su actividad económica cotidiana, deben llevar acciones filantrópicas o de acción social encaminadas a mejorar la calidad de vida de colectivos en situación de riesgo o debilidad.

Sin embargo, manifiesta (Hammnond, 2002) que en la actualidad casi las dos terceras partes de la población mundial son pobres, pero que la mayoría de las empresas compiten fuertemente por ofrecer productos y servicios al tercio restante que tiene el mayor poder adquisitivo.

En el Ecuador los emprendimientos, son un área de atención prioritaria para el gobierno del Ecuador, mismo que ha implementado políticas nacionales en busca del desarrollo del ecosistema emprendedor. El gobierno central para, equiparar las condiciones de acceso al emprendimiento urbano y rural, ha desarrollado dos programas en primero manejado por el Ministerio de Coordinación de la Producción, Empleo y Competitividad que apoya a ciudadanos a la creación de negocios con potencial de crecimiento, innovadores o altamente diferenciados.

En perspectiva de acondicionar un escenario adecuado para desarrollar emprendimientos, el gobierno del Ecuador incorporó las Finanzas Populares y Solidarias en su Constitución 2008, conforme establecen los artículos 308 al 312.

De acuerdo al informe generado por el (INEC, 2012), en un registro actualizado de las unidades económicas que producen bienes y servicios en el país, en donde se analiza las empresas desde su clasificación por sociedades y personas naturales, a nivel de empresas y establecimientos, permitiendo la identificación, localización, estratificación y demografía de las mismas.

Las provincias con mayor número de empresas son: Guayas con 58.754 empresas lo cual representa el 32.67\%, seguido de Pichincha con 50.269 que representa el $27.95 \%$, luego se encuentra Manabí 9.970 lo que representa el 5.54\%, inmediatamente esta Azuay con 8.438 lo que representa el $4.69 \%$ y al final se encuentra El Oro con 7.567 lo cual representa el $4.20 \%$.

De los datos antes citados, se identifican las provincias de alta concentración económica, lo cual dinamiza la economía de diversos sectores del país, es importante tener en cuenta que los emprendimientos forman parte de estos datos estadísticos.

Por otra parte de acuerdo a los resultados publicados por el (GEM, 2010), de los estudios realizados en varios países del Mundo, manifiestan que el Ecuador consta entre los 15 países con mayor tendencia a iniciar nuevos negocios. Las fortalezas de sus emprendedores son la juventud y la capacidad de detectar oportunidades del mercado; sus debilidades, la falta de capital y la poca capacitación. El emprendimiento puede ser un motor de la economía, pero su impacto aún es incipiente.

Según el estudio, el Índice de Actividad Emprendedora Temprana, conocido como TEA, es del 15,82\% en Ecuador. Esto quiere decir que siete de cada 10 ecuatorianos adultos están en proceso de iniciar un negocio o gestionando una nueva empresa (de no 
más de 42 meses). Pese al dinamismo que evidencian los números, este índice considerado el eje del GEM-ha registrado un importante descenso respecto del 2004, la primera vez que se aplicó el estudio localmente, cuando el TEA fue del 27,2\%, y ubicó al Ecuador en el tercer lugar del ranking mundial.

Una arista interesante en el análisis del emprendimiento son las motivaciones. La investigación demuestra que los emprendedores ecuatorianos que buscan una oportunidad de mercado doblan a aquellos que emprenden por necesidad; de este segmento, más de la mitad busca mayor independencia y el incremento de ingresos. Además, buena parte de estos nuevos negocios nacen en el sector de servicios al consumidor y se enfocan principalmente en el mercado doméstico.

Ecuador consta entre los 15 países con mayor tendencia a iniciar nuevos negocios. Las fortalezas de sus emprendedores son la juventud y la capacidad de detectar oportunidades del mercado; sus debilidades, la falta de capital y la poca capacitación. El emprendimiento puede ser un motor de la economía, pero su impacto aún es incipiente.

Según el estudio, el Índice de Actividad Emprendedora Temprana, conocido como TEA, es del 15,82\% en Ecuador. Esto quiere decir que siete de cada 10 ecuatorianos adultos están en proceso de iniciar un negocio o gestionando una nueva empresa (de no más de 42 meses). Pese al dinamismo que evidencian los números, este índice considerado el eje del (GEM, 2013) ha registrado un importante descenso respecto del 2004, la primera vez que se aplicó el estudio localmente, cuando el TEA fue del 27,2\%, y ubicó al Ecuador en el tercer lugar del ranking mundial.

Más de la mitad de los emprendedores ecuatorianos están entre los 25 y 44 años de edad y la incorporación de mujeres a este segmento es cada vez más significativa. La diferencia radica en que los hombres emprenden primordialmente por oportunidad, mientras que las mujeres son más sensibles a la necesidad. Esta motivación usualmente es más determinante en segmentos que no han tenido la oportunidad de acceder a educación formal o que han completado un nivel medio.(GEM, 2013)

Los datos antes citados, conducen a formular las siguientes preguntas: ¿De qué forma la RSE motiva el emprendimiento en el Ecuador? ¿Bajo qué paradigma de pensamiento administrativo se desarrollan los emprendimientos en el Ecuador? ¿Cómo incentivan las leyes del estado a los emprendimientos a desarrollar programas de RSE?

Para responder a estos cuestionamientos es necesario, se citarán varios autores que abordan el desarrollo de la empresa en la sociedad, para ello (Druker, 2001), analiza los principales cambios de la sociedad, la superación de del Estado Nación, y el nuevo significado del conocimiento así como el nuevo significado del conocimiento, así como los desafíos que esas transformaciones suponen en términos de oportunidades y amenazas.

Por otra parte aborda la significación social de la empresa que inmerso en este concepto se encuentra el emprendimiento, los principales retos que plantea el futuro para identificar quienes son los responsables de la conducción de las empresas, por ello es importante analizar el directivo de la empresa desde su comportamiento ético. (Kliksberg, 2006), en su libro "Ética para empresarios", en el cual habla de las grandes desigualdades del mundo respecto a variables sociales, para lograr emprendimientos socialmente responsables, se debe incorporar buenas prácticas, que nazcan de los 
procesos de la cadena de valor de los emprendimientos, lo que quiere decir es que se debe desarrollar emprendimientos basados en el paradigma interaccionista que desarrolle una visión incluyente y social de los actores en los emprendimientos, identificando los clientes, proveedores y competidores (stakeholders).

Solo identificando a los stakeholders, los emprendedores pueden identificar las expectativas de los diferentes grupos de interés, para a través de ello implementar Buenas Prácticas que mejoren las condiciones de los emprendimientos, pese a sus recursos limitados, se pueden desarrollar programas de RSE.

En la publicación de la revista (LIDERES, 2015), resalta que en el Ecuador más del 90\% de las empresas son familiares, Sylvia Cabrera de Uribe, directora ejecutiva del Instituto Ecuatoriano de Gobernanza Corporativa, cuenta que en Ecuador cada vez existe mayor conciencia sobre la responsabilidad familiar en las empresas, porque de ello depende la sostenibilidad de los negocios. Ella añade que la principal inquietud que tienen las empresas es cómo seleccionar a los miembros para el directorio.

Adicionalmente publicó (LIDERES, 2015), "Fabrizio Noboa, director del USFQ Business School, sostiene que en el Ecuador las organizaciones familiares son renuentes a profesionalizar a la empresa o a usar herramientas como protocolos o directorios. "El reto es para la nueva generación de una empresa familiar, que se está formando en el exterior". En las empresas del Grupo Godoy, con base en Loja, por ejemplo, la tercera generación ya empieza a participar. Este conglomerado tiene negocios en la elaboración y comercialización de alimentos, producción de envases de vidrio, una inmobiliaria y productos de arcilla. Amada Godoy, representante del grupo, asegura que la estrategia es sencilla: "observamos y respetamos las jerarquías en las empresas de la familia.

Respetamos las decisiones de cada hermano, sin importar que sea menor o mayor". Otro de los secretos es la equidad en el paquete accionario. "Cada socio tiene la misma participación". La equidad permite, según Godoy, generar riqueza para todos. Una sugerencia más que hace la representante de este grupo, cuyo primer negocio nació hace 40 años, es trabajar con reglas y documentos que faciliten el desempeño de los negocios. "También se necesita una cultura empresarial para que los familiares se consideren parte de la empresa como colaboradores. Y que esté claro que si un familiar tiene competencias, puede asumir nuevos cargos".

Finalmente, las empresas en el Ecuador han surgido de emprendimientos, que a pasos pequeños como manifiestan los entrevistados, van estructurando estrategias de RSE, enfocadas en los Stakeholders.

\section{Conclusiones}

La perspectiva funcionalista de la administración se caracteriza por su limitada capacidad crítica y el enfoque en la acumulación del capital.

La perspectiva interaccionista se enfoca en la administración idiosincrática y administración interpretativa, las dos enfatizan la dimensión grupal u holística buscando una administración integradora y administración dialógica, que busque el bienestar de las personas. 
El GEM sostiene que el emprendimiento puede ser un motor de la economía, pero su impacto aún es incipiente por la falta de enfoque integral de gestión que marque una estrategia de desarrollo y sostenibilidad en el tiempo.

Una de las herramientas para el desarrollo y la sostenibilidad organizacional es la RSE, que por su naturaleza se puede convertir en una estrategia de desempeño para alcanzar la supervivencia y calidad de los emprendimientos, una vez que se hayan entendido las particularidades de estas organizaciones.

\section{Referencias bibliográficas}

Benno, Z. (1996). GESTIÓN EDUCATIVA Y CALIDAD DE VIDA. En G. e. Latina:. Washington DC: El troquel.

Bilbao, a, \& Pachano, S. (2002). Rasgos y actitudes de los emprendedores. Proyecto Andino de Competitividad. from http://www.cid.harvard.edu/archive/andes/documents/workingpapers/razgosyactitu des/rasgos_actitudes_venezuela_competitiva.pdf

Coleman, J. S. (1990). Fundamentos de teoría social. (C. de I. Sociológicas, Ed.) (Primera en). Madrid- España. Retrieved from https://books.google.com/books?hl=es\&lr=\&id=Hev3oZ1vqSkC\&pgis=1

Drakopoulou Dodd, S., \& Hynes, B. C. (2011). The impact of regional entrepreneurial contexts upon enterprise education. Entrepreneurship \& Regional Development, (December 2012), 1-26. http://doi.org/10.1080/08985626.2011.566376

Druker, P. (2001). La productividad del trabajador del conocimiento. Máximo desafío 2001. Harvard Business Review, Deusto2, p. 16. California. Retrieved from http://www3.mapfre.com/estudios/boletin/N3/pdfs/productividad.PDF

Friedman, M. (1966). The Methodology of Positive Economics*. Retrieved November 30, 2015, from http://www.sfu.ca/ dandolfa/friedman-1966.pdf

GEM, E. (2013). GEM. Retrieved October 16, 2015, from http://www.espae.espol.edu.ec/images/documentos/publicaciones/libros/reportegem201 3.pdf

Izquierdo, R. J. S., \& Vicedo, J. C. (2009). La Responsabilidad Social Empresarial en un contexto de crisis. Repercusión en las Sociedades Cooperativas. CIRIEC-España, Revista de Economía Pública, Social Y Cooperativa, (65), 7-31. Retrieved from http://www.ciriec-revistaeconomia.es/banco/6501_Server_y_Capo.pdf

Kliksberg, B. (2006). Ética Empresarial: ¿Moda o Demanda Imparable? Boletín

Bimestral De La Fundación Carolina, 4, 1-5.

LIDERES, E. C.-. (2015). Las empresas se integran a la responsabilidad social corporativa | Revista Líderes. Retrieved November 30, 2015, from http://www.revistalideres.ec/lideres/empresas-integran-responsabilidad-socialcorporativa.html

Orrego, C. I. (2008). La Dimensión Humana Del Emprendimiento. Revista Ciencias Estratégicas, 16(20), 225-235. Retrieved from http://www.redalyc.org/articulo.oa?id=151312829001

Toca, C. (2017). Contributions to Social Responsibility, 393-408. Retrieved from http://www.revistas.unam.mx/index.php/rmcpys/issue/view/4553/showToc

Vives, A. (2013). Empleo y emprendimiento como responsabilidad social de las empresas. Journal Globalization, Competitiveness and Governability, 7(3), 16-33. http://doi.org/10.3232/GCG.2013.V7.N3.01 